\title{
NANO-ENGINEERED COATINGS ON TITANIUM IMPLANTS
}

\author{
Dikova Ts. \\ Department of Material Science, Faculty of Dental Medicine, \\ Medical University of Varna
}

\begin{abstract}
The present paper deals with the latest developments in nanoscale coatings on titanium implants. The main problems of dental implants and the main advantages of nanotechnologies are discussed. Precisely defined structures commensurable with the bone structure can be created on the implant surfaces using various nanotechnologies. Nanostructured coatings improve bioactivity and biocompatibilty influencing the whole process of osteoblast integration.
\end{abstract}

Key words: implants, implants coatings, nanotechnology, nano-engineered coatings

\section{INTRODUCTION}

Dental implantology is a field of dentistry rapidly advancing in recent years. Nowadays it is mainly used in esthetic dentistry, however, in the near future it will become a part of the general orthopedic dentistry as it offers new techniques for dental prosthesis. Using dental implantology, the common three part bridge could be substituted by a single implant with a crown which is more favourable for the patient. The main problem of dental implants is their biocompatibility and osteoblast integration. Nanotechnologies could give the right solution.

The main purpose of the present paper is to review the latest developments in nano-scale coatings on titanium implants.

\section{Implants}

An implant is an artificial structure whose purpose is to replace or stabilize damaged body functions. It includes many different types such as hip and knee joints, spinal implants, dental implants, and stents. Medical implants have to satisfy functionality demands defined by the working environment-human body. They should have biomechanical properties comparable to those of autogenous tissues without any adverse effects (2). The principal requirements of any medical implants are corrosion resistance, biocompatibility, bioadhesion, biofunctionality, processability and availability. The disintegration of the implant material which weakens the implant is a consequence of corrosion which exerts a harmful effect on the surrounding tissues and organs. Biocompatibility is the ability of a material to function with an appropriate host response in a specific application. It can be achieved in several ways. Either an implant can be made from a biocompatible material and

Address for correspondence:

Ts. Dikova, Dept. of Material Science

Faculty of Dental Medicine, Medical University of Varna

55 Marin Drinov Str., BG-9002 Varna, Bulgaria

e-mail: tsanka_dikova@abv.bg then implanted directly. Otherwise, biocompatibility can be achieved by coating the implant with a biocompatible material, or modifying the surface in another way.

\section{Materials for implants}

A number of biocompatible materials are used nowadays for implant production: titanium, stainless steel, porcelain, bioceramics, alumina alloys and various polymers. Metallic biomaterials can be divided into four subgroups: stainless steels, cobalt-based alloys, titanium metals and miscellaneous others (including tantalum, gold and other special metals) $(2,5,12)$. They are very effective in binding fractured bones, do not corrode and do not release harmful toxins when exposed to body fluids and, therefore, can be left inside the body for a long period of time. Their disadvantage is a much larger hardness and stiffness compared to the bone and possibility of interfering with bone regrowth.

Currently, implants are comprised mainly of titanium and titanium alloys $(2,12)$. The pure titanium has a biologically inert surface, which makes it biocompatible. So, it does not trigger unwanted immune reactions. It possesses excellent mechanical properties and very good osseointegration. Good corrosion resistance of titanium depends upon the formation of a solid oxide layer $\left(\mathrm{TiO}_{2}\right)$ to a depth of $10 \mathrm{~nm}$. Geometry, roughness and other characteristics of the implant surface importantly influence on the surface-tissue interaction, too. Implant surface can be modified by different ways (13). Mechanical methods for surface treatment can be divided into methods involving removal of surface material by cutting, abrasive action (grinding and polishing) and blasting by particles. Chemical methods include solvent cleaning, wet chemical etching and passivation treatments while electric polishing and anodic oxidation or anodizing belongs to the electrochemical surface methods.

\section{Implants coatings}

The using of coatings on the titanium implants improves their surface properties such as wear and corrosion resis- 
tance. The wettability, roughness and surface charge of the coated surfaces stimulate osteoblast adhesion and enhance matrix mineralization compared to the passive titanium surfaces. These coatings should have a sufficiently high adherence to the substrate throughout the range of conditions to which the implant is exposed in service. Such surface modification could be done by various processes $(12,13)$ : precipitations from the chemical vapour phase, Sol-Gel coatings, chemical vapour deposition (CVD), or physical vapour deposition (PVD).

The concept of bioactive coatings uses a principle of enabling the interfacial chemical bond between the implant and the bone tissue due to a specific biological response (9). One of the most popular bioactive coatings is hydroxyapatite (HA). It is similar to the mineral phase of natural hard tissue. HA can also be regarded as non-resorbable in a physiological environment, as long as it remains crystalline and of high purity (2). However, it has weaker mechanical properties and low resistance to fatigue failure.

\section{Nanotechnology}

Nowadays 'nanotechnology' means the design, characterization, production and application of structures, devices and systems by controlling shape and size at the nanometer scale $(14,18)$. 'Nanomaterial' is a material with one dimension less than $100 \mathrm{~nm}(0,1 \mu \mathrm{m})$, or in the range of $1-100 \mathrm{~nm}$. These dimensions are commensurable with the average atomic and molecular sizes of commonly used materials. Because the material's properties depend on their sizes, in the nano-scale, materials will possess specific properties which will influence on their physical, chemical and biological behaviour. The nano-scale particles have a very large surface in comparison with their volume and, consequently, a higher surface energy which makes them more active $(1,14)$.

Nano-scale particles and devices are commensurable with the cell structure. Bone is the most obvious example of a natural nanophase material. From the materials' point of view, hard tissues can be determined to be a composite material. Bone and teeth are composed of a mineral phase (carbonated HA) and an organic phase, mainly collagen and some other non-collagenous proteins. In the natural bone, collagen type-I forms fibrils with an interfibrillar space of $68 \mathrm{~nm}$ and depth of $35 \mathrm{~nm}$. HA crystals are embedded in fibrils and have an average size of $50 \times 25 \times 4 \mathrm{~nm}^{3}$ $(11,17)$. In the hard dental tissue, the difference is only in the quantitative proportion between inorganic and organic phase - about $97 \%-3 \%$ in the enamel, whereas in dentin this correlation is $70 \%-30 \%$, respectively.

The commensurability of the nanomaterials with the cell structures and their specific properties give new approach in the prevention, diagnosis and medication of diseases, thus determining many new opportunities for application in medicine and dental medicine as well (6).

\section{Nanotechnologies in implant production}

The design of implants could be discussed in the following four aspects: macro-design, mini-design, micro-design, and nano-design. The last two aspects which refer to the surface roughness at micro-level and to the molecular organization of the implant surface at nano-level are important for us. The application of nanotechnology to implants allows the structure or surface of a biomaterial to be precisely defined thus improving biocompatibilty. The main aspects in application of nanotechnology for implant development are three.

The first one concerns the bulk nanostructured materials usually titanium, its alloys and stainless steels, which possess higher strength, fatigue strength and corrosion resistance $(10,7)$.

The surface nanostructuring is another direction for development. Nanostructured surfaces can improve cellular attachment and direct the cells to grow into defined structures $(5,8)$.

Usage of nanocoatings for biocompatibility enhancement, decreasing the toxicity by increasing the corrosion resistance and better interaction with the surrounding cell

\section{Nano-engineered coatings}

In order to understand the topographic effect on the biological response of the coatings it is important to be familiar with their structure. It is formed at nano- and micro-level in the course of different technological processes. The increase of micro-roughness affects mainly the cellular response by means of cell morphological adaptation to the topography of the substrate, whereas nano-roughness that increases real surface area could have a major influence on the protein adsorption and ionic interactions.

The addition of nano-polymorphic features (comprising of tuft-like, plate-like, and nodular structures smaller than 100 $\mathrm{nm}$ ) to the micro-roughened surface of the Ti-6Al-4V samples (20) significantly increases the implant fixation, the area of periimplant bone formation and the percentage of bone-implant contact at the early and late healing stages. The hierarchical hybrid micro/nano-textured surfaces have been produced by acid etching and anodization (22). The addition of nanotubes to the microstructured surface enhances multiple osteoblast behaviors, resulting in better in vivo osseointegration. The presence of a vertically aligned $\mathrm{TiO}_{2}$ nanotube surface on titanium foils exerts a critical effect and improves the proliferation and mineralization of osteoblasts (15). The cell response depends on the nanotubes' diameter. It has been established that a diameter of $70 \mathrm{~nm}$ is the optimal size for $\mathrm{TiO}_{2}$ nanotube implants to obtain favourable osteoconductivity and osseointegration $(16,21)$. Furthermore, $\mathrm{TiO}_{2}$ nanotubes can control cell fate and interfacial osteogenesis by altering their nano-scale dimension.

The $\mathrm{TiO}_{2}$ nanotube surface can mimic the dimensions of the components of natural bone because the latter is composed of nanophase HA in the collagen matrix. It is well-known that the anatase phase $\mathrm{TiO}_{2}$ is much more efficient in nucleation and growth of HA than the rutile phase $\mathrm{TiO}_{2}$ because of the better lattice match with $\mathrm{HA}$ phase $(17,21)$. That is why the tendency in oxidation is to produce surfaces completely covered with anatase phase $\mathrm{TiO}_{2}$ 
which enhances the osseointegrative characteristics of the titanium $(15,16,21)$.

Coatings consisting of nanomatarials based on $\mathrm{TiO}_{2}, \mathrm{ZrO}_{2}$ and containing $\mathrm{Ag}, \mathrm{Zn}$ and $\mathrm{Cu}$ ions not only improve the osseointegration but also additionally exert an antimicrobial effect (19). Calcium phosphate nano-coatings improve the adhesion to hard tissues and favour bone growth (3). The bone cells grow faster and produce more calcium when the titanium implant is anodized and coated with carbon nano-tubes (4).

\section{CONCLUSION}

Precisely defined structures, commensurable with the bone structure are created on the implant surfaces using various nanotechnologies. Different materials are used for implants nano-coatings. The cell interfacial osseogenesis can be controlled by coating of $\mathrm{TiO}_{2}$ nanotubes. Surfaces coated with nano-HA ensure both good cell adhesion and enhanced protein synthesis and adsorption. Nanostructured coatings improve bioactivity and biocompatibilty influencing on the whole process of osteoblast integration.

\section{REFERENCES}

1. Aitken, R. J., M. Q. Chaudhry, A. B. Boxall, M. Hull. Manufacture and use of nanomaterials: current status in the UK and global trends.- Occup. Med., 56, 2006, No 5, 300-306.

2. Balazic, M., J. Kopac. Review: titanium and titanium alloy applications in medicine.- Int. J. Nano Biomaterials, 1, No 1, 2007, 3-34.

3. Bigi, A., M. Fini, B. Bracci, E. Boanini, P. Torricelli, G. Giavaresi, et al. The response of bone to nanocrystalline hydroxyapatite-coated Ti13Nb11Zr alloy in an animal model.- Biomaterials, 9, 2008, No 11, 1730-1736.

4. Brown University. Bone-growing nanomaterial could improve orthopaedic implants. Science Daily, 2007, Sep. 18. doi: http://www.sciencedaily.com

5. de Groot, R., J. Loeffler, U. Sutter. Roadmap Report. Concerning the use of nanomaterials in the medical \& health sector. Steinbeis-Europa-Zentrum, Sixth Framework Programme, 2006.

6. Dikova, Ts., M. Milkov. Nanomaterials in dental medicine.- Nanoscience \& Nanotechnology. E. Balabanova, I. Dragieva, eds. Sofia, BAS, NCCNT, 2009, 203-209.

7. Estrin, Y. Nanostructured materials for permanent and bioresorbable medical implants. $\mathrm{http} / / /$ www.azonano.com/details.asp?ArticleId $=2453$

8. Fasasi, A. Y., S. Mwenifumido, N. Rahbar, J. Chen, M. Li, A. C. Beye, et al. Nano-second UV laser processed micro-grooves onTi6Al4V for biomedical applications.- Mat. Sci. Eng. C, 29, 2009, No 1, 5-13.

9. Hench, L. L., R. J. Splinter, W. C. Allen, T. K. Greenlee. Bonding mechanisms at the interface of ceramic prosthetic materials.- J. Biomed. Mater. Res., 5, 1971, No 6, 117-141.

10. Kolobov, Y. Development and application of the implants based on nanostructured titanium with bioactive coatings.- In: International Scientific Conference "Rusnanotech '09". Moscow, 2009. www.rusnanoforum.ru (in Russian).

11. Landis, W. J., K. J. Hodgens, J. Arena, M. J. Song, B. F. McEwen. Structural relations between collagen and mineral in bone as determined by high voltage electron microscopic tomography.Microsc. Res. Tech., 33, 1996, No 2, 192-202.

12. Liu, X., P. K. Chu, C. Ding. Surface modi?cation of titanium, titanium alloys, and related materials for biomedical applications.- Mater. Sci. Eng. Rep., 47, 2004, 49-121.

13. Lutjering, G., J. C. Williams. Titanium. $2^{\text {nd }}$ ed. Berlin, Heidelberg, New York, Springer, 2007.

14. Maynard, A. Nanotechnology, overview and issues.- In: Nanotechnology: toxicological issues and environmental security. Dordrecht, Springer, 2007, $1-14$.

15. Oh, S., C. Daraio, L. H. Chen, T. R. Pisanic, R. R. Fiñones, S. Jin. Significantly accelerated osteoblast cell growth on aligned $\mathrm{TiO}_{2}$ nanotubes.- $J$. Biomed. Mater. Res. A, 78, 2006, No 1, 97-103.

16. Oh, S., K. S. Brammer, Y. S. Li, D. Teng, A. J. Engler, S. Chien, et al. Stem cell fate dictated solely by altered nanotube dimension.- Proc. Natl. Acad. Sci USA, 106, 2009, No 7, 2130-2135.

17. Rho, J. Y., I. Kuhn-Speating, P. Zioupos. Mechanical properties and the hierarchical structure of bone.- Med. Eng. Phys., 20, 1998, No 2, 92-102.

18. Royal Society. Nanoscience and nanotechnologies: opportunities and uncertainties. Summary and Recommendations, 2004. www.royalsoc.ac.uc

19. Search International Patent Applications. Nanomaterial coatings for osteointegrated biomedical prostheses. FR2007/001363, A61K 8/46. http://www.wipo.int

20. Ueno, T., N. Tsukimura, M. Yamada, T. Ogawa. Enhanced bone-integration of alkali- and heat-treated nanopolymorphic titanium in micro-to-nanoscale hierarchy.- Biomaterials, 32, 2011, No 30, 7297-7308.

21. Wang, N., H. Li, W. Lü, J. Li, J. Wang, Z. Zhang, et al. Effect of $\mathrm{TiO}_{2}$ nanotubes with different diameters on gene expression and osseointegration of implant in minipigs.- Biomaterials, 32, 2011, No 29, 6900-6911.

22. Zhao, L., S. Mei, P. K. Chu, Y. Zhang, Z. $\mathrm{Wu}$. The influence of hierarchical hybrid micro/nano-textured titanium surface with titania nanotubes on osteoblast functions.- Biomaterials, $\mathbf{3 1}$, 2010, No 19, 5072-5082. 